\title{
Cheap Talk Games with Two-Senders and Different Modes of Communication ${ }^{+}$
}

\author{
Esra E. Bayindir ${ }^{1}$, Mehmet Y. Gurdal ${ }^{2}$, Ayca Ozdogan ${ }^{3}$ and Ismail Saglam ${ }^{3, *}$ \\ 1 Hamburg Center for Health Economics, University of Hamburg, Esplanade 36, 20354 Hamburg, Germany; \\ esraerenb@gmail.com \\ 2 Department of Economics, Bogazici University, Bebek, Istanbul 34342, Turkey; mehmet.gurdal@boun.edu.tr \\ 3 Department of Economics, TOBB University of Economics and Technology, Sogutozu Cad. 43, Sogutozu, \\ Ankara 06560, Turkey; aozdogan@etu.edu.tr \\ * Correspondence: isaglam@etu.edu.tr \\ † We thank Santiago Sánchez-Pagés for sharing with us the software codes for a single sender-receiver game, \\ which we have modified and used in our experiments. We also acknowledge that this research was \\ supported by TOBB University of Economics and Technology. The usual disclaimer applies.
}

Received: 17 January 2020; Accepted: 1 April 2020; Published: 9 April 2020

\begin{abstract}
This paper deals with the effects of different modes of communication in a costless information transmission environment with multiple senders. To this aim, we present a theoretical and experimental study of three Cheap Talk games, each having two senders and one receiver. The communication of senders is simultaneous in the first, sequential in the second and determined by the receiver in the third game (the Choice Game). We find that the overcommunication phenomenon observed with only one sender becomes insignificant in our two-sender model regardless of the mode of communication. However, as to the excessive trust of the receiver, our results are not distinguished from those in the one-sender model. Regarding the Choice Game, our logistic regressions on experimental results suggest that the receiver is more likely to select simultaneous play if the previous play was simultaneous and the receiver earned the high payoff and much more likely to select simultaneous play if the messages were nonconflicting.
\end{abstract}

Keywords: strategic information transmission; truth-telling; trust; sender-receiver game

JEL Classification: C72; C90; D83

\section{Introduction}

A recent experimental literature analyzes information transmission in a class of sender-receiver games in which the only equilibrium is babbling; i.e., communication is not informative. In these games, a sender privately observes Nature's realization of a conflicting payoff table that could be of two equally likely types. The sender then transmits a message involving the type of the payoff table to the receiver, whose action will in turn determine an outcome in the payoff table chosen by Nature. The possible strategies are telling the truth and lying about the payoff table from the viewpoint of the sender whereas trusting and distrusting from the viewpoint of the receiver. For these games, it is known since the seminal work of Crawford and Sobel [1] that the sender will optimally not transmit any information in any sequential equilibrium. While the comparative statics predictions of this theory was experimentally confirmed by Dickhaut et al. [2], several experiments conducted in the last 
decade presented conflicting evidence, indicating overcommunication by the sender and overtrust by the receiver. ${ }^{1}$

In this paper, we study the effects of different modes of communication in a costless information transmission environment with two senders. Basically, we aim to identify whether the overcommunication and overtrust phenomena persist and which mode could potentially bring more benefit to an uninformed receiver. Absolutely, the possible welfare effects of introducing additional sender(s), under different modes of communication, to a single sender-receiver model might have implications from the perspective of mechanism design. Besides, communication with multiple senders is observed in real-life settings not very uncommonly. People often seek a second opinion especially when they think that the experts may have conflicting interests or biases for any reason. For example, when a patient sees a doctor for a serious sickness which may or may not require an operation, they usually would like to get an opinion of another doctor. Furthermore, both of the doctors may have conflicting interests and they may want to perform an operation regardless of the condition. There are several dimensions that may affect the aggregation of information by the patient. For example, should the patient tell the diagnosis of the first doctor to the second and then get the second doctor's advice or should she get a second opinion without revealing the advice of the first doctor to the second? Moreover, if she chooses to receive the advice of the second doctor after telling the suggestion of the first one, who should she believe if she receives conflicting messages? As another example, we can think of a customer who is shopping for an electronic product. Would it better for him to consult only one salesperson before making a decision to buy or could he benefit from the advice of an additional salesperson if he believes that both of them are trying to maximize the number of products that they sell? If he seeks a second opinion, should he inform the second salesperson about the advice of the first? Our study attempts to answer questions of these sort.

In our paper, we consider three different sender-receiver games played by two senders and one receiver, namely the Simultaneous, Sequential, and Choice Game. The informational setup in each game is similar to that in the single sender-receiver game studied by Sánchez-Pagés and Vorsatz [4]. The receiver only knows the possible payoff tables, whereas the two senders also know the actual payoff table. Each game is a constant-sum; so the receiver and the two senders as a whole have opposing interests. Additionally, we assume that the two senders' payoffs are always equal in order to isolate the effect of the order of play in the sequential communication of the senders with the receiver. In the Simultaneous Game, the two senders simultaneously transmit a payoff-relevant message (the type of the actual payoff table) to the receiver. In the Sequential Game, the two senders are named by sender 1 and sender 2 with respect to a given order, and then sender 1 transmits a payoff-relevant message that is received by both sender 2 and the receiver. Next, sender 2 transmits a payoff-relevant message to the receiver. Finally, in the Choice Game, the receiver first decides whether the Simultaneous or Sequential Game will be played, and then the chosen game is played accordingly. In each of these three games, the receiver takes an action after observing the message of the senders, and consequently the payoffs of the three players are determined by the actual payoff table chosen by Nature and the action taken by the receiver. Since preferences of the senders and the receiver are not aligned, the theory predicts that rational and self-interested senders will optimally not transmit any information under any mode of communication; and consequently the choice of the game will be immaterial for a rational receiver.

The main result we obtain from our experiments is that the excessive truth-telling phenomenon-which has been observed in sender-receiver games that involve a single sender but otherwise have similar structures-becomes insignificant (but also still persistent) in the presence of a second sender regardless of the mode of communication of the senders. On the other hand, the excessive trust the receiver is experimentally known to exhibit when she faces a single sender is not affected by the presence of a second sender under any type of communication. To investigate

1 See, for example, [3-8] among others. 
the determinants of the observed receiver behavior in the Choice Game we run logistic regressions. Among various results, we show that the receiver is more likely to select simultaneous play if the previous play was simultaneous and the receiver earned the high payoff and much more likely to select simultaneous play if the messages were nonconflicting additionally. ${ }^{2}$

The theoretical literature has studied the multi-sender cheap talk games quite well. ${ }^{3}$ Very recently, a number of models in this rapidly growing literature were also tested by game-theoretic laboratory experiments. The main focus of this literature has been to study the effect of different institutions on information transmission or to find conditions which ensure that a fully-revealing equilibrium exists. To the best of our knowledge, this literature is currently missing multi-sender extensions of one sender and one receiver models with an essentially unique and babbling equilibrium under different modes of communication. ${ }^{4}$

The basic models of cheap talk with essentially unique babbling equilibria have drawn attention in the experimental literature as these models enables one to clearly distinguish between the experimental observations and theoretical predictions. For example, Sánchez-Pagés and Vorsatz [4] show that when conflicting preferences in a baseline game of the described class are zero-sum but not too unequal, the subjects in the role of a sender transmit a correct message significantly more frequently than theoretically expected. They study the behavioral basis of the observed overcommunication by considering a punishment game. ${ }^{5}$ Their result is supported by [7], where a baseline sender-receiver game is played both under a sanction-free institution and under a sanctioning institution, where the receiver has the option to reduce the payoffs of both players to zero. ${ }^{6}$

More recently, Minozzi and Woon [23] analyze the effect of having a second sender in a sequential cheap talk game taking into account differences in alignment of preferences of the senders and receiver. They show that the receiver gets the same amount of information-which is higher that the theoretical prediction-with the addition of the second sender regardless of the alignment or competition between the senders.

Our model investigates a case with two senders (where the essentially unique equilibrium is babbling) to experimentally identify the effects of different modes of communication on the truth-telling of the senders and the trust of the receiver. The rest of the paper is organized as follows: Section 2 introduces two senders and one receiver games and presents some theoretical predictions. Section 3 presents the experimental design and procedures, while Section 4 reports experimental results. Section 5 contains several logistic regressions to estimate the receiver behavior in the Choice Game. Finally, Section 6 provides some discussion and concluding remarks. (The instructions corresponding to the experimental games are presented in Appendix A, and the proofs of all the propositions are relegated to Appendix B).

2 Also, in order to explain the observed differences in behavior of senders and receiver in simultaneous and sequential plays, we use a logit agent quantal response equilibrium (logit-AQRE) model, following [7] and [8]. We present the maximum likelihood estimation results of this model in the online appendix at: http:/ /isaglam.etu.edu.tr/BGOS-2020Online-Appendix.pdf.

3 For example, [9-13] among others extend the basic one sender and one receiver model in Crawford and Sobel [1] by allowing two perfectly informed senders. [14-16] consider the case with two imperfectly informed senders while $[15,16]$ also analyze the effects of alternative communication modes, namely simultaneous and sequential transmission of information. A common feature of these extensions is that the policy space is unidimensional, while [17-22] consider multidimensional models of cheap talk.

4 See $[23,24]$ for experimental models studying communication with multiple senders.

5 An alternative behavioral explanation for excessive truth-telling is provided by Sánchez-Pagés and Vorsatz [5] using their baseline and punishment games in [4] with a modification that the sender in the baseline game additionally has a costly option of remaining silent. They show that overcommunication in the baseline game can be attributed to lying aversion.

6 Several papers have also studied the robustness of overcommunication phenomenon. For example, Peeters et al. [25] show that overcommunication of the sender disappears in the presence of rewards, whereas the trust by the receiver increases significantly. Gurdal et al. [8] analyze the robustness of excessive truth-telling and trust to the intervention of an honest regulator (equivalently to the presence of non-strategic sender types). They show that excessive truth-telling and excessive trust are higher under intervention than under the absence of intervention. 


\section{The Model and the Theoretical Predictions}

We extend the sender-receiver game studied by Sánches-Pagés and Vorsatz [4] by adding a second sender to the environment. We denote sender 1 , sender 2 and receiver by $S_{1}, S_{2}$ and $R$, respectively. At the beginning of the game, Nature chooses a payoff table $A$ or $B$ (see Table 1 ) with equal probability that determines the final payoffs (in TL) of the three players.

Table 1. Payoff Tables.

\begin{tabular}{cccc}
\hline Table A & Sender 1 & Sender 2 & Receiver \\
\hline Action U & 4.5 & 4.5 & 1 \\
Action D & 0.5 & 0.5 & 9 \\
\hline Table B & Sender 1 & Sender 2 & Receiver \\
\hline Action U & 0.5 & 0.5 & 9 \\
Action D & 4.5 & 4.5 & 1 \\
\hline
\end{tabular}

The senders are privately informed about the realized payoff table. Depending on the information observed, $S_{1}$ and $S_{2}$ respectively choose possibly mixed actions $p$ and $q$ from the set of messages $M=\{A, B\}$. Here, $p$ and $q$ denote the probabilities that the message $A$ is submitted by $S_{1}$ and $S_{2}$, respectively. After observing the messages submitted by the two senders, the receiver chooses a possibly mixed action $r$ from the set of actions $\{U, D\}$, showing the probability that $U$ is played by the receiver. We analyze two games that differ with respect to the mode of communication of the senders with the receiver, namely the Simultaneous and the Sequential Game. In the Simultaneous Game, the two senders simultaneously transmit a message to the receiver after observing the actual state. Then, the receiver takes an action knowing that the senders have not observed each others' messages. In the Sequential Game, first moves sender 1, transmitting a message. Then, after observing the message of sender 1 , sender 2 transmits a message. Knowing that sender 2 has observed the message transmitted by sender 1 , the receiver takes an action that determines the payoffs of all three players. The third game we consider is the Choice Game, where the receiver moves first and chooses whether the Simultaneous or the Sequential Game is going to be played, and then the chosen game is played accordingly.

\subsection{The Simultaneous Game}

In the Simultaneous Game, both senders have two information sets corresponding to the events that the actual payoff table is $A$ or $B$. When the actual state is $A$, the strategies of $S_{1}$ and $S_{2}$ are $p_{A}$ and $q_{A}$, respectively denoting the probabilities that sender 1 and sender 2 choose message $A$ when the actual state is $A$. Similarly, when the actual state is $B$, the strategies of $S_{1}$ and $S_{2}$ are $p_{B}$ and $q_{B}$, respectively denoting the probabilities that sender 1 and sender 2 choose message $A$ when the actual state is $B$. The receiver, on the other hand, has four information sets corresponding to four possible message pairs that can be submitted by the two senders. Here, $r_{A A}, r_{A B}, r_{B A}$ and $r_{B B}$ denote the probabilities that action $U$ is played corresponding to the observed messages of $S_{1}$ and $S_{2}$ (which are denoted in the subscripts of $r$ in order). The receiver forms the beliefs $\mu_{A A}, \mu_{A B}, \mu_{B A}$, and $\mu_{B B}$, each denoting the belief that the actual state is $A$ after observing the corresponding set of messages by $S_{1}$ and $S_{2}$ specified in the subscripts, respectively.

Proposition 1. Any sequential equilibrium of the Simultaneous Game satisfies

$$
\begin{aligned}
& p_{A}=p_{B}=p \in[0,1] ; \\
& q_{A}=q_{B}=q \in[0,1] ;
\end{aligned}
$$

with the supporting belief system is $\mu_{i j}=\frac{1}{2}$ for every $i j \in\{A A, A B, B A, B B\}$ on the equilibrium path. 
Corollary 1. The probability of observing an untruthful message by any of the senders in any sequential equilibrium is $1 / 2$.

This says that no information is revealed in any equilibrium. Sender 1 plays $B$ when the true state is $A$ with probability $\left(1-p_{A}\right)$ and choose $A$ when the true state is $B$ with probability $p_{B}$. As each state is equally likely and $p_{A}=p_{B}$ in any equilibrium, it is straightforward that the receiver expects to see an untruthful message from $S_{1}$ with probability one half. The same argument is true for the messages of sender 2 .

Remark 1. The receiver's strategies should satisfy the following condition in order to have $p_{A}=p_{B}=p>0$ and $q_{A}=q_{B}=q>0$ as a sequential equilibrium:

$$
p=\frac{r_{B B}-r_{B A}}{r_{A A}-r_{A B}+r_{B B}-r_{B A}} \quad \text { and } \quad q=\frac{r_{B B}-r_{A B}}{r_{A A}-r_{A B}+r_{B B}-r_{B A}}
$$

These conditions imply that $r_{A A}>r_{A B}, r_{A A}>r_{B A}, r_{B B}>r_{A B}$ and $r_{B B}>r_{B A}$ in any equilibrium where the senders use completely mixed strategies. ${ }^{7}$

\subsection{The Sequential Game}

In the Sequential Game, sender 1 has two information sets, whereas sender 2 has four information sets. The strategies of $S_{1}$ when the actual state is $n \in\{A, B\}$ is denoted by $p_{n}$ as before. The strategies of $S_{2}$ (i.e., the probability that message $A$ is chosen) when the actual state is $n \in\{A, B\}$ and the sender 1 has communicated message $i \in\{A, B\}$ is denoted by $q_{n}(i)$. The receiver, again, has four information sets, at which $r_{A A}, r_{A B}, r_{B A}$ and $r_{B B}$ are the probabilities that action $U$ is played corresponding to the observed messages of $S_{1}$ and $S_{2}$, denoted in the subscripts of $r$, respectively. The receiver forms the beliefs $\mu_{i j}$ showing the probability that the actual state is $A$ after observing the message $i \in\{A, B\}$ from sender 1 and $j \in\{A, B\}$ from sender 2 .

Proposition 2. In any sequential equilibrium of the Sequential Game,

$$
\begin{aligned}
p_{A} & =p_{B}=p \in[0,1] \\
q_{A}(A) & =q_{B}(A)=q_{1} \in[0,1] \\
q_{A}(B) & =q_{B}(B)=q_{2} \in[0,1]
\end{aligned}
$$

with the supporting belief system $\mu_{i j}=\frac{1}{2}$ for $i j \in\{A A, A B, B A, B B\}$ on the equilibrium path.

Corollary 2. The probability of observing an untruthful message by any of the senders in any sequential equilibrium is $\frac{1}{2}$.

As $S_{1}$ plays $B$ when the true state is $A$ with probability $\left(1-p_{A}\right)$ and choose $A$ when the true state is $B$ with probability $p_{B}$, it is straightforward that the receiver expects to see an untruthful message by $S_{1}$ with probability one half. The expected probability of seeing an untruthful message by $S_{2}$ is given by the following expression:

$$
\frac{1}{2}\left[\left(1-p_{A}\right)\left(1-q_{A}(B)\right)+p_{A}\left(1-q_{A}(A)\right)\right]+\frac{1}{2}\left[p_{B} q_{B}(A)+\left(1-p_{B}\right) q_{B}(B)\right]
$$

which is also equal to $1 / 2$ in any equilibria.

7 For instance, $p=\frac{3}{4}, q=\frac{3}{4}$ and $r_{A A}=\frac{1}{3}, r_{B B}=\frac{1}{2}, r_{A B}=r_{B A}=\frac{1}{4}$ constitute an equilibrium. 


\subsection{The Choice Game}

Since the equilibria of the Simultaneous Game and the Sequential Game induce the same expected payoff to the receiver, she should be indifferent choosing between the two games. After the receiver's choice of the communication mode, one of the equilibria of the chosen game is played according to the requirements of sequential rationality.

\subsection{Hypotheses}

Based on the predictions of the model, we obtain several hypotheses regarding the truth-telling levels of the senders and trust levels of the receivers.

Hypothesis 1. (Truth-telling in the Simultaneous Game). Both senders will tell the truth with 50\% probability in the Simultaneous Game.

Hypothesis 2. (Truth-telling in the Sequential Game) Both senders will tell the truth with 50\% probability in the Sequential Game and truth-telling by Sender 2 will be independent of the observed message of Sender 1.

Hypothesis 3. (Trust in the Simultaneous Game) Upon observing a non-conflictive message, the receiver will trust with 50\% probability in the Simultaneous Game.

Hypothesis 4. (Trust in the Sequential Game) Upon observing a non-conflictive message, the receiver will trust with $50 \%$ probability in the Sequential Game.

Hypothesis 5. (Receiver Behavior in the Choice Treatment) The receiver will choose each of the Simultaneous and Sequential Games with 50\% probability.

\section{Experimental Design and Procedures}

All experimental sessions were conducted in the Social Sciences Laboratory at TOBB University of Economics and Technology during 28-30 March 2012. We sent a school wide invitation e-mail to undergraduate students informing that for the invited experiment they could register online for a date and time they choose. Those who registered also received reminder e-mails 1 day before the session. In total, the experiment was conducted over 8 sessions, one with 8 subjects the rest with 12 subjects. We had 92 subjects in total and each session lasted abut 55-60 min. We performed our experiments with the computer software z-Tree developed by Fischbacher [26].

Our design is a modification of the setup used in $[4,5,25]$. Each session consisted of three treatments which we term as the Simultaneous Treatment, the Sequential Treatment and the Choice Treatment. The order of these treatments during a session could be either Simultaneous-Sequential-Choice or Sequential-Simultaneous-Choice. Each treatment lasted 12 periods. Before the experiment began, subjects were randomly assigned to groups of 4 and there were 23 groups in total. At the start of each period, two of these 4 subjects were assigned sender roles, one was assigned the receiver role and one was assigned the observer role. ${ }^{8}$ During the 12 periods in a given treatment, each subject played 6 times as a sender, 3 times as a receiver and 3 times as an observer. The order of role assignments was randomly determined. ${ }^{9}$

8 We consider the observer role so as to check whether our subjects, when they are neither the receiver nor a sender, are able to make correct guesses about the outcomes of the games.

9 Given that the subjects make choices in the same group for 36 periods, it may be a valid concern that anonymity may have been somewhat disregarded as the repetition could have an effect in the experiment even though the roles are randomly assigned in each period within a group. Since all treatments may be affected to some extent by the repetition, we believe that the differences in treatments are not due to the repetition effect. Moreover, our experimental results indicate that the behavior of the players are not significantly different than the theoretical predictions of the one-shot game in most of the cases (see Section 4). As the choice of running sessions with groups of 4 is essentially done for using non-parametric tests 
In the Simultaneous Treatment, subjects played the following game for each period: First, subjects learned about their role assignments for that period which could either be sender 1, sender 2, the receiver or the observer. Afterwards, sender 1 and sender 2 were informed about the true state (the payoff table being played) which could be either "Table A" or "Table B". Following this, sender 1 and sender 2 simultaneously and without seeing each other's decision, decided on the message they want to send to the receiver. The messages could be either "The payoff table is A" or "The payoff table is $\mathrm{B}^{\prime \prime}$. The observer, on the other hand, was also informed about the payoff table and chose one of the following guesses: "The receiver will earn 9 and sender 1 and sender 2 will each earn 0.5 " or "The receiver will earn 1 and sender 1 and sender 2 will each earn $4.5^{\prime \prime}$. Next, the receiver was informed about the messages of sender 1 and sender 2 on the same screen and was asked which payoff table she thinks is more likely to be the correct one. Then the receiver choose among two possible actions: " $\mathrm{U}$ " or " $\mathrm{D}$ ". After this choice of action, the payoffs were realized accordingly and a summary of the period was shown to the senders, the receiver and the observer. For the senders and the receiver, this summary includes information about the true state, the signals sent, the belief of the receiver, the action chosen by the receiver and the payoffs to the senders and to the receiver. For the observer the summary includes her guess, the earnings of the receiver and the senders and her own earning. If her guess was correct, the observer earned $5 \mathrm{TL}$ for that period and if not $0 \mathrm{TL}$.

The Sequential Treatment differs from the above setup in the way the senders acted. In this treatment, sender 1 first chose the message to be sent and then this was showed first to sender 2, who in turn chose her message to be sent. The rest of the game is similar. In the Choice Treatment on the other hand, the receiver acted first and chose the way she preferred the messages to be sent. In particular, for each period, the subject with the receiver role chose if she wants to play the game as in the Simultaneous Treatment or the game as in the Sequential Treatment. Following this choice, the game corresponding to the choice of the receiver was played.

After the three treatments were finished, subject answered several questions about their choices during the experiment. Following this, payments were displayed on the subject's screen. Each subject was paid the sum of her average earnings in the Simultaneous Treatment, average earnings in the Sequential Treatment and average earnings in the Choice Treatment plus a participation fee of 5 TL. Average total earnings (including the participation fee) were $14.26 \mathrm{TL}$ and at the time of the experiment, 1 TL corresponded to 0.6325 USD.

\section{Experimental Results}

92 subjects in our experiment constituted 23 distinct groups. In the following three subsections (Sections 4.1-4.3) we calculate the percentage of the variables of interest (truth-telling, trust, non-conflicting messages, truthfulness of non-conflicting messages etc.) for all distinct groups and use these independent observations in our analysis. Numerical values reported in the tables correspond to the mean value of the associated variable across all distinct groups. Below, we start with describing the sender behavior.

\subsection{Senders}

Considering all three treatments in the experiment, we find that the mean percentage of truthful messages per group is close to $53 \%$. However, it is not significantly above the theoretical prediction of $50 \%$ ( $p$-value is 0.057 in a Wilcoxon signed-rank test). Although this is consonant with the previous studies finding that subjects in general tell the truth more often than predicted, termed as overcommunication, we see that adding a second sender overshadows this phenomenon.

for the receiver behavior in the Choice Treatment, our estimation finding (Result 4 in Section 5) in that regard shows that the receiver values the information acquired in the previous period more than the information acquired in all other past periods in the Choice Treatment. These results imply that the learning effect due to repetition must be minimal. 
In Table 2, we summarize the behavior of senders in plays where they act simultaneously. The two columns respectively show sender behavior under all plays in the Simultaneous Treatment and plays in the Choice Treatment where receivers preferred the senders to play simultaneously. ${ }^{10}$ Senders exhibit excessive truth-telling in the Simultaneous Treatment by sending truthful messages with a frequency of 54\%; however, we fail to reject Hypothesis 1 ( $p$-value is 0.083 in a Wilcoxon signed-rank test). Furthermore, senders nearly randomize between truth and lie in the Choice Treatment in plays where the receiver prefers simultaneous messages.

Under simultaneous mode of communication, the two senders' agreement frequency is above $50 \% .{ }^{11}$ Furthermore, with a frequency of $58.2 \%$, the non-conflicting messages in the Simultaneous Treatment are considerably more likely to be truthful than the theoretical prediction of $50 \%$ ( $p$-value is 0.054 in a Wilcoxon signed-rank test).

Table 2. Sender Behavior with Simultaneous Message s ${ }^{a}$.

\begin{tabular}{lcc}
\hline & Simultaneous Treatment & Choice Treatment \\
\hline $\begin{array}{l}\text { \% Sender is truthful } \\
\% \text { Senders are non-conflictive }\end{array}$ & $54.0^{*}$ & $49.0^{*}$ \\
$\%$ Non-conflicting messages & $58.2^{* *}$ & $53.4^{* *}$ \\
are correct & & $50.2^{*}$ \\
\hline $\mathrm{N}$ & 23 & 23 \\
\hline
\end{tabular}

Sender behavior when the two senders act sequentially is summarized in Table $3 .{ }^{12}$ The first column reports sender behavior under all plays in the Sequential Treatment, the second column reports plays in the Choice Treatment where receivers preferred the senders to play sequentially. Our results are somewhat in line with Hypothesis 2 about truth telling. In the Sequential Treatment, while sender 1 perfectly randomizes, sender 2 tells the truth with a probability higher than $50 \%$ that is not significantly different than the theoretical prediction. However, truth-telling by Sender 2 is not independent of the earlier message of Sender 1 . The probability with which sender 2 is truthful given that sender 1 lies is $58.9 \%$ compared to $54 \%$ when sender 1 is truthful (and in the Choice Treatment where the receivers choose sequential mode of communication, this figure goes up to $63 \%$ ). Hence, the contribution to the excessive truth-telling in sequential plays comes from sender 2.

We see that non-conflicting messages are observed less frequently in the Sequential Treatment $(46.4 \%)$ than in the Simultaneous Treatment (51.4\%). The lower frequency of non-conflicting messages in sequential plays is mainly due to the fact that the subjects in the role of sender 2 have a tendency to revert the message when sender 1 lies. Moreover, non-conflicting messages are more likely to be truthful in the Simultaneous Treatment (58.2\%) thanin the Sequential Treatment (54.5\%).

10 Looking at the 276 instances during the Choice Treatment, we see that the receivers preferred simultaneous messages in 152 cases $(55 \%)$ and sequential messages in 124 cases $(45 \%)$.

11 The theoretical predictions for the probabilities in the first and last rows are $1 / 2$, whereas the theoretical prediction for the probability that the two senders' messages are non-conflictive in simultaneous plays is $p_{A} q_{A}+\left(1-p_{A}\right)\left(1-q_{A}\right) \in[0,1]$.

12 The theoretical predictions for the probabilities in the first two rows and the last row are $1 / 2$. The theoretical predictions for the probabilities in all the remaining rows are arbitrary in the interval $[0,1]$. To see this, one can check that the probability that sender 2 is truthful when sender 1 is truthful is $p_{A} q_{A}(A)+\left(1-p_{B}\right)\left(1-q_{B}(B)\right)$. Similarly, the probability that sender 2 is truthful when sender 1 lies is $\left(1-p_{A}\right) q_{A}(B)+p_{B}\left(1-q_{B}(A)\right)$. One can also check that the probability that senders are non-conflictive is $p_{A} q_{A}(A)+\left(1-p_{A}\right)\left(1-q_{A}(B)\right)$. 
Table 3. Sender Behavior with Sequential Messages ${ }^{a}$.

\begin{tabular}{|c|c|c|}
\hline & Sequential Treatment & Choice Treatment \\
\hline$\%$ Sender is truthful & $53.3 *$ & $52.3 *$ \\
\hline$\%$ Sender 1 is truthful & $50.0 *$ & $51.6 *$ \\
\hline $\begin{array}{l}\% \text { Sender } 2 \text { is truthful when } \\
\text { sender } 1 \text { is truthful }\end{array}$ & $54.0^{* *}$ & $48.0^{* *}$ \\
\hline $\begin{array}{l}\text { \% Sender } 2 \text { is truthful when } \\
\text { sender } 1 \text { lies }\end{array}$ & $58.9^{* *}$ & $63.0^{* *}$ \\
\hline$\%$ Senders are non-conflictive & $46.4^{* *}$ & $45.9^{* *}$ \\
\hline $\begin{array}{l}\% \text { Non-conflicting messages } \\
\text { are correct }\end{array}$ & $54.5^{*}$ & $62.5^{*}$ \\
\hline $\mathrm{N}$ & 23 & 23 \\
\hline
\end{tabular}

\subsection{Receivers}

Prior to choosing their action, receivers in our experiment were asked to state their beliefs. This belief elicitation stage wasn't incentivized and each receiver was asked which payoff table she thinks is more likely to be the correct one (answering $A, B$, or equally likely). We focus on the cases where the messages by two senders are non-conflictive, and in Table 4 we present the frequency of beliefs that are in line with non-conflictive messages. The theoretical prediction for this frequency is $50 \%$ in all treatments. As Table 4 shows, this prediction holds true in the Sequential Treatment as well as in the Choice Treatment (with sequential or simultaneous messages). However, in the Simultaneous Treatment, the stated beliefs agree with the non-conflictive messages of senders $59.2 \%$ of the time and this frequency is significantly above $50 \%$.

Table 4. Frequency of Beliefs in Line with Non-Conflicting Messages(\%) ${ }^{a}$.

\begin{tabular}{lclc}
\hline \multicolumn{2}{c}{ Simultaneous Messages } & \multicolumn{2}{c}{ Sequential Messages } \\
\hline Simultaneous Treatment & $59.2^{*}$ & Sequential Treatment & $52.3^{*}$ \\
Choice Treatment & $50.7^{*}$ & Choice Treatment & $48.6^{*}$ \\
\hline N & 23 & & 23
\end{tabular}

${ }^{a}$ In the first and second columns, observations under the Choice Treatment only include cases where receivers preferred the senders to act simultaneously and sequentially, respectively. The values that are significantly different from $50 \%$ are given in bold. * The theoretical prediction is 50 .

A variable of particular interest is the receivers' trust frequency when the messages of the senders are non-conflictive. In the context of the game subjects played in the experiment, we define trust as choosing the optimal action by assuming that the non-conflictive messages of two senders is truthful. This corresponds to choosing action $D$ when both senders claim that the payoff table is $A$ and choosing action $U$ when both senders claim that the payoff table is $B$. The theoretical predictions for the frequencies of these two actions are respectively represented by $1-r_{A A}$ and $r_{B B}$ in all games we consider and found to be arbitrary in $[0 \%, 100 \%]$. On the other hand, our experimental results in Table 5 show that the receiver's trust frequency is generally above $50 \%$ regardless of the way messages were sent.

In Table 5, we observe that the average of the fraction of trusted messages per group is $56.5 \%$ for the Simultaneous Treatment and 61.3\% for plays in the Choice Treatment where receiver preferred simultaneous messages. Although the latter value is remarkably above 50\%, we cannot reject Hypothesis 3 ( $p$-value is 0.069 in a Wilcoxon signed-rank test). When messages were sent sequentially, the average of the fraction of trusted messages per group is $59.6 \%$ for the Sequential Treatment and $55.1 \%$ for plays in the Choice Treatment where receiver preferred sequential messages. The first 
one of these two values is significantly above $50 \%$ ( $p$-value is 0.036 in a Wilcoxon signed-rank test), refuting Hypothesis 4.

Table 5. Receiver Trust Frequency (\%) ${ }^{a}$

\begin{tabular}{lrlc}
\hline \multicolumn{2}{c}{ Simultaneous Messages } & \multicolumn{2}{c}{ Sequential Messages } \\
\hline Simultaneous Treatment & $56.5^{*}$ & Sequential Treatment & $59.6^{*}$ \\
Choice Treatment & $61.3^{*}$ & Choice Treatment & $55.1^{*}$ \\
\hline $\mathrm{N}$ & 23 & & 23 \\
\hline
\end{tabular}

${ }^{a}$ In the first and second columns, observations under the Choice Treatment only include cases where receivers preferred the senders to act simultaneously and sequentially, respectively. The values that are significantly different from $50 \%$ are given in bold. ${ }^{*}$ The theoretical prediction is arbitrary in $[0,100]$.

In Table 6, we summarize the preferences of receivers over game type in the Choice Treatment. We observe that the receivers preferred the Simultaneous mode of communication slightly more often, but the difference between the choice frequencies is not statistically significant, in line with Hypothesis 5.

Table 6. Receiver Behavior in the Choice Treatment.

\begin{tabular}{cc}
\hline $\begin{array}{c}\text { Number of Times Simultaneous } \\
\text { Messages Is Preferred }\end{array}$ & Number of Subjects \\
\hline 0 & 22 \\
1 & 18 \\
2 & 22 \\
3 & 30 \\
\hline
\end{tabular}

\subsection{Observers}

We summarize the behavior of observers in Table 7, which presents the mean fraction of guesses per group that the outcome of the play will be favorable for the receiver (i.e., the receiver earns $9 \mathrm{TL}$ and senders earn 0.5 TL each) as well the mean fraction of correct guesses per group.

In the Simultaneous and Sequential Treatments, subjects are more likely to guess that the outcome of the play will be favorable for the senders. Contrary to this, in the Choice Treatment, subjects' guesses shift to the other direction in plays where the receiver preferred simultaneous messages ( $p$-value is 0.058 in a Wilcoxon signed-rank test). Subjects' guesses are more likely to be wrong than correct during plays in the Choice Treatment where the receiver preferred sequential messages ( $p$-value is 0.075 in a Wilcoxon signed-rank test).

Table 7. Observer Behavior.

\begin{tabular}{lcc}
\hline & $\begin{array}{c}\text { \% Guesses of Favorable } \\
\text { Outcome for the Receiver }\end{array}$ & $\begin{array}{c}\text { \% Correct } \\
\text { Guesses }\end{array}$ \\
\hline Simultaneous Treatment & $44.2^{*}$ & $45.7^{*}$ \\
Sequential Treatment & $48.2^{*}$ & $48.2^{*}$ \\
Choice Treatment (Simultaneous) & $59.1^{*}$ & $51.2^{*}$ \\
Choice Treatment (Sequential) & $55.2^{*}$ & $44.5^{*}$ \\
\hline $\mathrm{N}$ & 23 & 23 \\
\hline \multicolumn{2}{c}{ * The theoretical prediction is 50.}
\end{tabular}

To summarize our results, the experimental findings do not provide support to refute the first three of our hypotheses, although the truth-telling levels and trust levels are generally above $50 \%$ in both games. On the other hand, the frequency of receivers' trust to non-conflicting messages in 
the Sequential Treatment is significantly different than the theoretical prediction of one half, refuting Hypothesis 4. Moreover, Sender 1 perfectly randomizes between truth telling and lies with 50\% and the behavior of Sender 2 seems to be highly dependent on the behavior of Sender 1 in the Sequential Game. In particular, Sender 2 is more likely to tell the truth when Sender 1 lies compared to when Sender 1 tells the truth. It seems that the overcommunication phenomenon disappears for senders acting as the first movers in sequential plays. Furthermore, in line with Hypothesis 5, the receiver chooses in the Choice Treatment the two modes of communication with (almost) equal probabilities. In the next two sections, we will try to explore the factors underlying the observed behavior of the subjects in our experiments.

\section{Logistic Analysis for the Choice Treatment}

Here, we estimate the determinants of the observed receiver behavior in the Choice Treatment. Table 8 reports the logistic regression results. The dependent variable is the simultaneous choice in all of the specifications. In the first specification, we consider the effects of receivers' average payoffs over the simultaneous and sequential plays, updated in the Choice Treatment. Average payoffs do not have a statistically significant effect on the choice probability. For the second specification, we find that receiver is 2.43 times more likely to select simultaneous play in cases where the previous play was simultaneous and the receiver earned the high payoff than in cases where the previous play was sequential and the receiver earned the low payoff. The receiver is $53 \%$ less likely to select simultaneous play if the previous play was sequential and the receiver earned the high payoff relative to the case in which the previous play was sequential and the receiver earned the low payoff. The probability of choosing simultaneous play when the previous play was simultaneous and the receiver earned the low payoff is not statistically significantly different from the case where the previous play was sequential and the receiver earned the low payoff.

Estimation Result 1. The receiver is (i) more likely to select simultaneous play if the previous play was simultaneous and (ii) much more likely to select simultaneous play if the previous play was simultaneous and the receiver earned the high payoff relative to the case in which the previous play was sequential.

Table 8 also shows that ratio of nonconflicting messages in the Simultaneous Treatment has a positive effect on the probability of selecting simultaneous choice. In our estimations, we have used the ratio of nonconflicting messages in the simultaneous and sequential treatments as a control, being representative of the groups' cooperative tendencies or abilities to coordinate. These establish the following.

Estimation Result 2. High ratio of nonconflicting messages in both the Simultaneous and the Sequential Treatment increases the likelihood of simultaneous choice in the Choice Treatment; however the estimated impact is much larger in the Simultaneous Treatment than in the Sequential Treatment.

In the first, fifth, sixth and seventh specifications, the updated average payoffs of the receivers in the simultaneous and sequential plays are also included. Our estimations show that both variables are statistically insignificant at 95\% confidence level and the magnitudes of the coefficients are close to one, suggesting the following.

Estimation Result 3. The receiver values the information acquired in the previous period more than the information acquired in all other past periods.

In Table 8 we have also decomposed the results of the previous period depending on whether the messages were nonconflicting. The receiver is statistically significantly more likely to select simultaneous play if the previous play was simultaneous, the receiver earned the high payoff and the messages were nonconflicting than all other cases. Additionally, the receiver is statistically significantly less likely to select simultaneous play if the previous play was sequential and the receiver earned the 
high payoff than all other cases. Whether the messages were nonconflicting in sequential play does not statistically significantly affect the probability of choosing simultaneous play.

Estimation Result 4. If the previous play is simultaneous, the receiver is more likely to choose simultaneous play when the messages were nonconflicting and the receiver earned the high payoff relative to all other cases. On the contrary, if the previous play is sequential, whether the messages were conflicting or nonconflicting does not have a statistically significant effect on the probability of choosing simultaneous play and the receiver has the lowest probability of choosing simultaneous play when the previous play was sequential and the receiver earned the high payoff. 
Table 8. Logistic Analysis of Simultaneous Choice.

\begin{tabular}{|c|c|c|c|c|c|c|c|}
\hline & I & II & III & IV & $\mathbf{V}$ & VI & VII \\
\hline $\begin{array}{l}\text { Simwon }{ }^{\dagger} \text { ( } 1 \text { if previous play was simultaneous and } \\
\text { receiver earned high payoff) }\end{array}$ & & $\begin{array}{l}2.43 * * \\
(2.06)\end{array}$ & $\begin{array}{l}1.81 \\
(0.84)\end{array}$ & $\begin{array}{c}1.14 \\
(0.32)\end{array}$ & $\begin{array}{l}2.27 * \\
(1.92)\end{array}$ & $\begin{array}{l}1.72 \\
(0.8)\end{array}$ & $\begin{array}{c}1.12 \\
(0.28)\end{array}$ \\
\hline $\begin{array}{l}\text { Seqwon }{ }^{\dagger}(1 \text { if previous play was sequential and } \\
\text { receiver earned high pavoff) }\end{array}$ & & $0.47^{*}$ & $\begin{array}{c}0.68 \\
(-0.71)\end{array}$ & $0.43^{* *}$ & $\begin{array}{c}0.43^{*} \\
(-1.93)\end{array}$ & $\begin{array}{c}0.67 \\
(-0.74)\end{array}$ & $0.41^{* *}$ \\
\hline $\begin{array}{l}\text { Simlost }{ }^{\dagger} \text { ( } 1 \text { if previous play was simultaneous and } \\
\text { receiver earned low payoff) }\end{array}$ & & $\begin{array}{l}1.18 \\
(0.42)\end{array}$ & $\begin{array}{l}2.02 \\
(1.33)\end{array}$ & & $\begin{array}{l}1.08 \\
(0.21)\end{array}$ & $\begin{array}{l}1.90 \\
(1.24)\end{array}$ & \\
\hline $\begin{array}{l}\text { Simwonnc }{ }^{+}(1 \text { if previous play was simultaneous with } \\
\text { nonconflicting messages and receiver earned high payoff) }\end{array}$ & & & $\begin{array}{c}3.67 * * \\
(1.96)\end{array}$ & $\begin{array}{c}3.67 * * \\
(1.96)\end{array}$ & & $\begin{array}{l}3.70 * * \\
(2.04)\end{array}$ & $\begin{array}{l}3.68^{* *} \\
(2.04)\end{array}$ \\
\hline $\begin{array}{l}\text { Seqwonnc }{ }^{\dagger}(1 \text { if previous play was sequential with } \\
\text { nonconflicting messages and receiver earned high payoff) }\end{array}$ & & & $\begin{array}{c}0.99 \\
(-0.01)\end{array}$ & & & $\begin{array}{c}0.88 \\
(-0.24)\end{array}$ & \\
\hline $\begin{array}{l}\text { Simlostnc }{ }^{\dagger} \text { ( } 1 \text { if previous play was simultaneous with } \\
\text { nonconflicting messages and receiver earned low payoff) }\end{array}$ & & & $\begin{array}{c}0.72 \\
(-0.71)\end{array}$ & & & $\begin{array}{c}0.70 \\
(-0.77)\end{array}$ & \\
\hline $\begin{array}{l}\text { Seqlostnc }{ }^{\dagger}(1 \text { if previous play was sequential with } \\
\text { nonconflicting messages and receiver earned low payoff) }\end{array}$ & & & $\begin{array}{l}2.05 \\
(1.33)\end{array}$ & & & $\begin{array}{l}2.10 \\
(1.45)\end{array}$ & \\
\hline $\begin{array}{l}\text { Average payoff of the receivers over simultaneous plays, } \\
\text { updated in the Choice Treatment }\end{array}$ & $\begin{array}{c}0.97 \\
(-0.14)\end{array}$ & & & & $\begin{array}{c}0.95 \\
(-0.27)\end{array}$ & $\begin{array}{c}0.90 \\
(-0.59)\end{array}$ & $\begin{array}{c}0.91 \\
(-0.51)\end{array}$ \\
\hline $\begin{array}{l}\text { Average payoff of the receivers over sequential plays, } \\
\text { updated in the Choice Treatment }\end{array}$ & $\begin{array}{l}1.26 \\
(1.47)\end{array}$ & & & & $1.27 *$ & $1.26^{*}$ & $\begin{array}{c}1.26 \\
(1.61)\end{array}$ \\
\hline Ratio of nonconflicting messages in the Simultaneous & 8.88 & 2.88 & 2.59 & 2.53 & 5.26 & 4.73 & 4.68 \\
\hline Treatment & (1.6) & $(0.98)$ & $(0.95)$ & $(0.90)$ & $(1.33)$ & $(1.37)$ & $(1.28)$ \\
\hline Ratio of nonconflicting messages in the Sequential & 1.46 & 1.30 & 1.27 & 1.26 & 1.65 & 1.72 & 1.71 \\
\hline Treatment & $(0.33)$ & $(0.26)$ & $(0.22)$ & $(0.22)$ & $(0.49)$ & $(0.5)$ & $(0.50)$ \\
\hline Constant & $\begin{array}{c}0.11 \\
(-1.29)\end{array}$ & $\begin{array}{c}0.54 \\
(-0.76)\end{array}$ & $\begin{array}{c}0.40 \\
(-1.01)\end{array}$ & $\begin{array}{c}0.64 \\
(-0.59)\end{array}$ & $\begin{array}{c}0.14 \\
(-1.26)\end{array}$ & $\begin{array}{c}0.13 \\
(-1.21)\end{array}$ & $\begin{array}{c}0.19 \\
(-1.00)\end{array}$ \\
\hline Pseudo R2 & 0.015 & 0.063 & 0.086 & 0.079 & 0.072 & 0.095 & 0.088 \\
\hline
\end{tabular}

Dependent variable is simultaneous choice. Odds ratios are reported, $z$-values are reported in parenthesis. Standard errors are clustered at the group level. ${ }^{*}$ and ${ }^{* *}$ denote significance at the $90 \%$ and $95 \%$ confidence levels respectively. ${ }^{+}:=$Dummy variables. 


\section{Discussion and Concluding Remarks}

In order to identify the effects of different modes of communication in information transmission with multiple senders, we considered in this paper three versions of a constant-sum Cheap-Talk game played by two senders and one receiver, namely the Simultaneous, Sequential, and Choice Game. In the Simultaneous Game, the two senders simultaneously transmit a payoff-relevant message to the receiver. In the Sequential Game, first sender 1 transmits a payoff-relevant message that is received by both sender 2 and the receiver, and then sender 2 transmits a payoff-relevant message to the receiver. In the Choice Game, the receiver first decides which of the two games will be played. After observing the messages of the senders, the receiver next takes an action that determines the payoff of all.

Since the preferences of the two senders and the receiver are assumed to be conflictive, the theoretical prediction is that rational senders do not transmit any information under any mode of communication; hence a rational receiver is indifferent between the two modes of communication. To test this prediction, we conducted an experiment with eight sessions and 92 subjects in total. Each session consisted of three treatments that lasted 12 periods. The order of these treatments during a session could be either Simultaneous-Sequential-Choice or Sequential-Simultaneous-Choice. Before the experiment began, subjects were randomly assigned to groups of four and there were 23 groups in total. During the 12 periods in a given treatment, each subject played six times as a sender, three times as a receiver and three times as an observer, the order of which was randomly determined.

We can summarize our findings in the following three categories: (i) the overall behavior of subjects compared to the single-sender studies, (ii) the differences in the behavior of the senders in the Simultaneous and Sequential treatments, and (iii) the determinants of the receiver's behavior in the Choice treatment.

(i) We find that the excessive truth-telling phenomenon-which has been observed in sender-receiver games that involve a single sender but otherwise have similar structures-becomes insignificant but still persists in the presence of a second sender regardless of the mode of communication of the senders. On the other hand, in response to a non-conflicting pair of messages by the two senders the excessive trust of the receiver that is observed when she faces a single sender is not affected by the presence of a second sender under any type of communication.

(ii) Despite similar theoretical predictions, we find differences in the behavior of the senders in Simultaneous and Sequential Treatments. The senders exhibit excessive truth-telling by sending truthful messages with a frequency of $54 \%$ in the Simultaneous Game and 53.3\% in the Sequential Game. However, the frequency that sender 1 is truthful is $50 \%$ in the Sequential Game, whereas the frequency that sender 2 is truthful given that sender 1 is truthful is $54 \%$ and it is as high as $58.9 \%$ when sender 1 lies in the Sequential Game. Thus, the contribution to the excessive truth-telling comes from senders playing the second move in the Sequential Game. Moreover, senders send non-conflicting messages $51.4 \%$ of the time in Simultaneous Game and $46.4 \%$ in the Sequential Game. While non-conflicting messages in the Sequential Game are more likely to be truthful than not with a frequency of $54.5 \%$, this figure is remarkably higher in the Simultaneous Game with $58.2 \%$.

(iii) In the Choice Game, receivers prefer simultaneous messages slightly (but insignificantly) more often than sequential messages. We have estimated the determinants of the receiver behavior in the Choice Treatment using logistic regressions. We have found that the receiver is more likely to select simultaneous play if the previous play was simultaneous and the receiver earned the high payoff and much more likely to select simultaneous play if the messages were nonconflicting additionally. ${ }^{13}$

13 Also, the receiver is statistically significantly the least likely to select simultaneous play if the receiver earned the high payoff in a sequential play in the last period. Also, high ratio of nonconflicting messages in both the Simultaneous and the Sequential Treatment increases the likelihood of simultaneous choice in the Choice Treatment, while the estimated impact is larger in the Simultaneous Treatment than in the Sequential Treatment. 
As a modest policy implication of our results, we could say that if someone who gets advice from two experts (with conflicting interests to hers) merely aggregates the information she receives, it would be better for her to go with the simultaneous mode of communication. This is because it is more likely to observe nonconflicting messages in the Simultaneous Game, which are remarkably more likely to be truthful, and the receiver on average would be better off by just following the advice provided by this nonconflicting message. If she is receiving advice through the sequential mode of communication, she should disregard the information sent by the expert that is the first mover and follow the advice of the second mover (who knows the message sent by the first mover and thus whether he has been truthful or not).

In the current study, we have focused on a multi-sender Cheap Talk game with different modes of communication where the senders' information is perfect and their interests are perfectly aligned with each other while strictly conflicting with that of the receiver. Future research may study how the theoretical and experimental results would change if one relaxes any of these assumptions so as to capture a wider range of real life examples.

Author Contributions: Conceptualization, M.Y.G., A.O. and I.S.; methodology, M.Y.G., A.O. and I.S.; software, E.E.B., M.Y.G. and I.S.; validation, all authors; formal analysis, all authors; investigation, all authors; resources, M.Y.G.; data curation, E.E.B and M.Y.G; writing-original draft preparation, all authors; writing-review and editing, all authors; visualization, all authors; supervision, all authors; project administration, M.Y.G.; funding acquisition, M.Y.G. All authors have read and agree to the published version of the manuscript.

Funding: The experiments in this research were funded by TOBB University of Economics and Technology.

Conflicts of Interest: The authors declare no conflict of interest. Also, the funder had no role in the design of the study; in the collection, analyses, or interpretation of data; in the writing of the manuscript, or in the decision to publish the results.

\section{Appendix A. Instructions}

\section{Welcome!}

Thank you for your participation. The aim of this study is to understand how people decide in certain situations. From now on, talking to each other is prohibited. Violation of this rule requires immediate termination of the experiment. Please raise your hand to ask questions. This way, everybody will hear your question and our answer.

The experiment will be conducted through the computer and you will make all your decisions using the computer. Your earnings depend on your decisions as well as the decisions of other participants. These earnings and your participation fee will be paid to you in cash at the end of the experiment. The experiment consists of 3 different parts. We start with describing Part 1.

\section{Part 1}

In this part of the experiment you will play a game which will last 12 periods. Before the first period, the system will assign you to groups of 4 . These groups will remain the same throughout the experiment. A participant will only interact with participants from her own group but will not get to know the identity of other group members during or after the experiment.

Now, let's have a closer look at the game. Please do not hesitate to ask questions.

In the beginning of each period, 2 participants in your group will be assigned the sender roles, 1 participant will be assigned the receiver role and 1 participant will be assigned the observer role. At the end of 12 periods, each of you will have played 6 times as a sender, 3 times as a receiver and 3 times as an observer. The order of these role assignments is random.

During each period, after role assignments have been made, the system will choose one of the following: Table A or Table B. It is equally likely for the system to choose Table A or Table B. The earnings in that period will depend on the table chosen by the system and the choice of action $U$ or action $\mathrm{D}$ by the receiver. 
Table A1. Payoff Tables.

\begin{tabular}{cccc}
\hline Table A & G1 & G2 & Receiver \\
\hline Action U & 4.5 & 4.5 & 1 \\
Action D & 0.5 & 0.5 & 9 \\
\hline Table B & G1 & G2 & Receiver \\
\hline Action U & 0.5 & 0.5 & 9 \\
Action D & 4.5 & 4.5 & 1 \\
\hline
\end{tabular}

At each period, one of the senders in the group will be named as G1 and the other will be named as G2. These roles will be randomly assigned and G1 and G2 will earn the same amount for that period. For example, if the system chooses Table A and the receiver chooses action U, both G1 and G2 will earn $4.5 \mathrm{TL}$ and the receiver will earn $1 \mathrm{TL}$ for that period.

\section{Senders' task}

At the beginning of each period, G1 and G2 will be informed about the table chosen by the system for that period. G1 and G2 will make the first decisions of that period. This decision is the choice of the message to be delivered to the receiver and telling whether the system chose Table A or Table B. Since these messages are going to be sent simultaneously, no sender will get to know the message of the other sender. The senders are free to decide whether their messages are correct or not.

\section{Receiver's task}

The receiver will first see the messages of G1 and G2, but will not know the table chosen by the system. At the screen that the receiver observes these messages, she will be asked her belief about the actual table that will determine the payoffs for that round.

In the next screen, the receiver will choose action $U$ or action $D$.

After the receiver makes her choice, the earnings will be determined based on the actual table chosen by the system and the choice of the receiver.

\section{Observer's task}

The observer will guess what the earnings of the senders and the receiver will be in a given period. Due to the structure of the game, her guess could be one of the two types:

(1) Receiver: 9 TL; G1 and G2: 0.5 TL.

(2) Receiver: 1 TL; G1 and G2: 4.5 TL.

If her guess is correct, the observer will earn 5 TL for that period and 0 TL otherwise.

At the end of each period, a summary screen will provide information about the choices in that period and the earnings.

\section{Payment}

Based on your earnings for each period, your average earnings per period will be calculated. You can see this amount at the bottom of the summary screen. The average earnings at the end of period 12 will be your earnings from part 1 of the experiment.

Your total earnings in the experiment will be "earnings in part 1" + "earnings in part 2" + "earnings in part 3 " + "a participation fee of 5 TL".

\section{Part 2}

Now, we will start part 2 of the experiment. In this part of the experiment, you will play a game that will last for 12 periods. Your group and the payoff tables in this part will be the same as in the first part of the experiment. 
The new game is similar to the game used in the previous part of the experiment, but it has the following differences:

In this game, the sender chosen as G1 will first choose her message to the receiver and the other sender, G2, will see this message and then choose her own message. The receiver will see the messages of G1 and G2, and again she will not know the real payoff table chosen by the system.

The rest of the game is the same as in the previous part. The assignment of the roles G1 and G2 will be random as before.

\section{Part 3}

Now, we will start part 3 of the experiment. In this part of the experiment, you will play a game that will last for 12 periods. Your group and the payoff tables in this part will be the same as in the first part of the experiment.

But, during each period in this part of the experiment, the receiver will choose the way that the senders will convey their messages. In other words, the receiver will decide whether the senders will send their messages simultaneously or sequentially. As you may remember, these are the methods for sending messages used in the two parts of the experiment.

To summarize,

- If the receiver decides the messages to be sent simultaneously, both G1 and G2 will choose their messages at the same time, without seeing each other's messages.

- If the receiver decides the messages to be sent sequentially, first G1 will choose her message and then G2 will observe this message and choose her message.

The assignment of the roles G1 and G2 will be random as before.

\section{Appendix B. Proofs of Propositions}

Proof. (Proposition 1) First, we derive the best responses of the players at each information set. The best responses of $S_{1}$ after tables $A$ and $B$ have been observed are given as:

$$
\begin{gathered}
p_{A} \in\left\{\begin{array}{lll}
\{1\} & \text { if } & q_{A}\left(r_{A A}-r_{B A}\right)+\left(1-q_{A}\right)\left(r_{A B}-r_{B B}\right)>0 \\
{[0,1]} & \text { if } & q_{A}\left(r_{A A}-r_{B A}\right)+\left(1-q_{A}\right)\left(r_{A B}-r_{B B}\right)=0 \\
\{0\} & \text { if } & q_{A}\left(r_{A A}-r_{B A}\right)+\left(1-q_{A}\right)\left(r_{A B}-r_{B B}\right)<0
\end{array}\right. \\
p_{B} \in\left\{\begin{array}{lll}
\{1\} & \text { if } & q_{B}\left(r_{A A}-r_{B A}\right)+\left(1-q_{B}\right)\left(r_{A B}-r_{B B}\right)<0 \\
{[0,1]} & \text { if } & q_{B}\left(r_{A A}-r_{B A}\right)+\left(1-q_{B}\right)\left(r_{A B}-r_{B B}\right)=0 \\
\{0\} & \text { if } & q_{B}\left(r_{A A}-r_{B A}\right)+\left(1-q_{B}\right)\left(r_{A B}-r_{B B}\right)>0
\end{array}\right.
\end{gathered}
$$

On the other hand, the best responses of $S_{2}$ after tables $A$ and $B$ have been observed are as follows:

$$
\begin{array}{r}
q_{A} \in\left\{\begin{array}{lll}
\{1\} & \text { if } & p_{A}\left(r_{A A}-r_{A B}\right)+\left(1-p_{A}\right)\left(r_{B A}-r_{B B}\right)>0 \\
{[0,1]} & \text { if } & p_{A}\left(r_{A A}-r_{A B}\right)+\left(1-p_{A}\right)\left(r_{B A}-r_{B B}\right)=0 \\
\{0\} & \text { if } & p_{A}\left(r_{A A}-r_{A B}\right)+\left(1-p_{A}\right)\left(r_{B A}-r_{B B}\right)<0
\end{array}\right. \\
q_{B} \in\left\{\begin{array}{lll}
\{1\} & \text { if } & p_{B}\left(r_{A A}-r_{A B}\right)+\left(1-p_{B}\right)\left(r_{B A}-r_{B B}\right)<0 \\
{[0,1]} & \text { if } & p_{B}\left(r_{A A}-r_{A B}\right)+\left(1-p_{B}\right)\left(r_{B A}-r_{B B}\right)=0 \\
\{0\} & \text { if } & p_{B}\left(r_{A A}-r_{A B}\right)+\left(1-p_{B}\right)\left(r_{B A}-r_{B B}\right)>0
\end{array}\right.
\end{array}
$$


The receiver's best responses after observing a message $i j \in\{A A, A B, B A, B B\}$ depend on the beliefs formed at that information set:

$$
r_{i j} \in\left\{\begin{array}{lll}
\{1\} & \text { if } & \mu_{i j}<\frac{1}{2} \\
{[0,1]} & \text { if } & \mu_{i j}=\frac{1}{2} \\
\{0\} & \text { if } & \mu_{i j}>\frac{1}{2}
\end{array}\right.
$$

Furthermore, the beliefs, calculated by Bayes' rule (whenever possible), are as follows:

$$
\begin{aligned}
& \mu_{A A}=\frac{p_{A} q_{A}}{p_{A} q_{A}+p_{B} q_{B}}, \quad \mu_{A B}=\frac{p_{A}\left(1-q_{A}\right)}{p_{A}\left(1-q_{A}\right)+p_{B}\left(1-q_{B}\right)}, \\
& \mu_{B A}=\frac{\left(1-p_{A}\right) q_{A}}{\left(1-p_{A}\right) q_{A}+\left(1-p_{B}\right) q_{B}}, \quad \mu_{B B}=\frac{\left(1-p_{A}\right)\left(1-q_{A}\right)}{\left(1-p_{A}\right)\left(1-q_{A}\right)+\left(1-p_{B}\right)\left(1-q_{B}\right)} .
\end{aligned}
$$

We want to show that the senders use the same strategy at the two information sets. To arrive at a contradiction, we consider the following cases: (1) One of the senders uses different strategies, while the other sender uses the same strategy at the two information sets; (2) Both of the senders use different strategies at the two information sets.

Case 1: Suppose that $S_{1}$ uses different strategies, i.e., $p_{A} \neq p_{B}$; and, without loss of generality, let's assume $p_{A}>p_{B}$. First, consider the case $q_{A}=q_{B}=q \in(0,1)$. Then, the consistency of beliefs requires $\mu_{A A}>\frac{1}{2}, \mu_{A B}>\frac{1}{2}, \mu_{B A}<\frac{1}{2}$, and $\mu_{B B}<\frac{1}{2}$. The best responses of the receiver at each information set under these beliefs become $r_{A A}=0, r_{A B}=0, r_{B A}=1$, and $r_{B B}=1$. But then, $S_{1}$ 's best responses are $p_{A}=0$ and $p_{B}=1$, which contradicts to our hypothesis that $p_{A}>p_{B}$. Now, suppose that $q_{A}=q_{B}=q=0$. With these strategies, the beliefs become $\mu_{A B}>\frac{1}{2}$ and $\mu_{B B}<\frac{1}{2}$. Having these beliefs, the receiver's best responses can be found as $r_{A B}=0$ and $r_{B B}=1$. Then, the best responses of $S_{1}$ are $p_{A}=0$ and $p_{B}=1$, again contradicting to our hypothesis. Next, assume that $q_{A}=q_{B}=q=1$. Under these strategies (given the hypothesis $p_{A}>p_{B}$ ), the beliefs can be calculated as $\mu_{A A}>\frac{1}{2}$, and $\mu_{B A}<\frac{1}{2}$ with the associated best responses for the receiver being $r_{A A}=0$ and $r_{B A}=1$. This in turn suggests that the best responses of $S_{1}$ should be $p_{A}=0$ and $p_{B}=1$, which provides the desired contradiction to $p_{A}>p_{B}$.

Case 2: Suppose that $p_{A} \neq p_{B}$ and $q_{A} \neq q_{B}$. Without loss of generality, we assume that $p_{A}>p_{B} \geq 0$ and $q_{A}>q_{B} \geq 0$. Then, the beliefs can be calculated as $\mu_{A A}>\frac{1}{2}$ and $\mu_{B B}<\frac{1}{2}$. The best responses of the receiver at these information sets become $r_{A A}=0$ and $r_{B B}=1$. If $q_{A}<1$, for $p_{A}>0$ to be the best response of $S_{1}$, the best responses of the receiver should satisfy $r_{A A}=r_{B A}=0$ and $r_{A B}=r_{B B}=1$. But if $r_{A A}=r_{B A}=0$ and $r_{A B}=r_{B B}=1$, then $q_{A}=0$, which is a contradiction as $q_{A}>q_{B} \geq 0$, by assumption. If $q_{A}=1$, then for having $p_{A}>0$ as a part of equilibrium, the best response of the receiver should satisfy $r_{A A}=r_{B A}=0$. In turn, $q_{A}=1$ can be a best response to these strategies only if $r_{A B}=0$ and $p_{A}=1$ (in addition to $r_{A A}=r_{B A}=0, r_{B B}=1$ ). But, then $p_{B}$ equals to 1 if $q_{B}<1$ and $q_{B}$ equals to 1 if $p_{B}<1$, which is the desired contradiction (since by assumption $p_{B} \neq 1$ and $q_{B} \neq 1$ as $p_{B}<p_{A}$ and $q_{B}<q_{A}$ ).

Since the senders are symmetric we exclude the symmetric situations. In all the other cases, we get at least one of the beliefs different than $\frac{1}{2}$. The corresponding best responses of the receiver at such information sets are pure strategies; and, the best responses of the senders against these pure strategies give the desired contradiction unless the senders use the same strategies at each information sets. Also, when $p_{A}=p_{B} \in(0,1)$ and $q_{A}=q_{B} \in(0,1)$, the beliefs can be easily calculated as $\mu_{i j}=\frac{1}{2}$ and they can be assigned in a consistent way off the equilibrium path. 
Proof. (Proposition 2) The best response of $S_{1}$ after table $A$ is observed is as follows:

$$
p_{A} \in\left\{\begin{array}{lll}
\{1\} & \text { if } & q_{A}(A) r_{A A}+\left(1-q_{A}(A)\right) r_{A B}-q_{A}(B) r_{B A}-\left(1-q_{A}(B)\right) r_{B B}>0 \\
{[0,1]} & \text { if } & q_{A}(A) r_{A A}+\left(1-q_{A}(A)\right) r_{A B}-q_{A}(B) r_{B A}-\left(1-q_{A}(B)\right) r_{B B}=0 \\
\{0\} & \text { if } & q_{A}(A) r_{A A}+\left(1-q_{A}(A)\right) r_{A B}-q_{A}(B) r_{B A}-\left(1-q_{A}(B)\right) r_{B B}<0
\end{array}\right.
$$

The best response of $S_{1}$ after table $B$ is observed is as follows:

$$
p_{B} \in\left\{\begin{array}{lll}
\{1\} & \text { if } & q_{B}(A) r_{A A}+\left(1-q_{B}(A)\right) r_{A B}-q_{B}(B) r_{B A}-\left(1-q_{B}(B)\right) r_{B B}<0 \\
{[0,1]} & \text { if } & q_{B}(A) r_{A A}+\left(1-q_{B}(A)\right) r_{A B}-q_{B}(B) r_{B A}-\left(1-q_{B}(B)\right) r_{B B}=0 \\
\{0\} & \text { if } & q_{B}(A) r_{A A}+\left(1-q_{B}(A)\right) r_{A B}-q_{B}(B) r_{B A}-\left(1-q_{B}(B)\right) r_{B B}>0
\end{array}\right.
$$

The best response of $S_{2}$ when the actual table is $A$ and the sender 1 has sent message $A$ is given by:

$$
q_{A}(A) \in\left\{\begin{array}{lll}
\{1\} & \text { if } & r_{A A}-r_{A B}>0 \\
{[0,1]} & \text { if } & r_{A A}-r_{A B}=0 \\
\{0\} & \text { if } & r_{A A}-r_{A B}<0
\end{array}\right.
$$

The best response of $S_{2}$ when the actual table is $A$ and the sender 1 has sent message $B$ is given by:

$$
q_{A}(B) \in\left\{\begin{array}{lll}
\{1\} & \text { if } & r_{B A}-r_{B B}>0 \\
{[0,1]} & \text { if } & r_{B A}-r_{B B}=0 \\
\{0\} & \text { if } & r_{B A}-r_{B B}<0
\end{array}\right.
$$

The best response of $S_{2}$ when the actual table is $B$ and the sender 1 has sent message $A$ is given by:

$$
q_{B}(A) \in\left\{\begin{array}{lll}
\{1\} & \text { if } & r_{A B}-r_{A A}>0 \\
{[0,1]} & \text { if } & r_{A B}-r_{A A}=0 \\
\{0\} & \text { if } & r_{A B}-r_{A A}<0
\end{array}\right.
$$

The best response of $S_{2}$ when the actual table is $B$ and the sender 1 has sent message $B$ is given by:

$$
q_{B}(B) \in\left\{\begin{array}{lll}
\{1\} & \text { if } & r_{B B}-r_{B A}>0 \\
{[0,1]} & \text { if } & r_{B B}-r_{B A}=0 \\
\{0\} & \text { if } & r_{B B}-r_{B A}<0
\end{array}\right.
$$

The receiver's best response after observing message $i j \in\{A A, A B, B A, B B\}$ is given by:

$$
r_{i j} \in\left\{\begin{array}{lll}
\{1\} & \text { if } & \mu_{i j}<\frac{1}{2} \\
{[0,1]} & \text { if } & \mu_{i j}=\frac{1}{2} \\
\{0\} & \text { if } & \mu_{i j}>\frac{1}{2}
\end{array}\right.
$$

where the beliefs calculated by the Bayes' rule (whenever possible) are as follows:

$$
\mu_{A A}=\frac{p_{A} q_{A}(A)}{p_{A} q_{A}(A)+p_{B} q_{B}(A)}, \quad \mu_{B B}=\frac{\left(1-p_{A}\right)\left(1-q_{A}(B)\right)}{\left(1-p_{A}\right)\left(1-q_{A}(B)\right)+\left(1-p_{B}\right)\left(1-q_{B}(B)\right)}
$$




$$
\mu_{A B}=\frac{p_{A}\left(1-q_{A}(A)\right)}{p_{A}\left(1-q_{A}(A)\right)+p_{B}\left(1-q_{B}(A)\right)}, \quad \mu_{B A}=\frac{\left(1-p_{A}\right) q_{A}(B)}{\left(1-p_{A}\right) q_{A}(B)+\left(1-p_{B}\right) q_{B}(B)} .
$$

In the first step, we want to show that $S_{1}$ uses the same strategy at the two information sets. To do that, first, we are going to assume that $S_{2}$ uses the same strategies at her information sets, then we will allow for the case in which $S_{2}$ may use different strategies.

Case 1: Suppose that $S_{2}$ uses the same strategy $q_{A}(A)=q_{B}(A)$ and $q_{A}(B)=q_{B}(B)$. For a contradiction, without loss of generality, we assume that $p_{A}>p_{B}$.

Case 1.a: Assume that $q_{A}(A)=q_{B}(A) \in(0,1)$ and $q_{A}(B)=q_{B}(B) \in(0,1)$. Then, the beliefs can be calculated as $\mu_{A A}>\frac{1}{2}, \mu_{A B}>\frac{1}{2}, \mu_{B A}<\frac{1}{2}$, and $\mu_{B B}<\frac{1}{2}$. The associated best responses of the receiver are $r_{A A}=0, r_{A B}=0, r_{B A}=1$, and $r_{B B}=1$. The best responses of $S_{1}$ in turn become $p_{A}=0$ and $p_{B}=1$, which contradicts to our hypothesis.

Case 1.b: Now, assume that $q_{A}(A)=q_{B}(A)=0$ and $q_{A}(B)=q_{B}(B)=0$. Then, the beliefs become $\mu_{B B}<\frac{1}{2}$ and $\mu_{A B}>\frac{1}{2}$. The best responses of the receiver corresponding to these beliefs are $r_{B B}=1$ and $r_{A B}=0$. The best responses of $S_{1}$ in turn become $p_{A}=0$ and $p_{B}=1$, which again contradicts to our hypothesis $p_{A}>p_{B}$.

Case 1.c: Now, assume that $q_{A}(A)=q_{B}(A)=1$ and $q_{A}(B)=q_{B}(B)=1$. Then, the beliefs become $\mu_{B A}<\frac{1}{2}$ and $\mu_{A A}>\frac{1}{2}$. The best responses of the receiver corresponding to these beliefs are $r_{B A}=1$ and $r_{A A}=0$. The best responses of $S_{1}$ in turn become $p_{A}=0$ and $p_{B}=1$, which gives the desired contradiction.

Case 1.d: We next assume that $q_{A}(A)=q_{B}(A)=0$ and $q_{A}(B)=q_{B}(B) \in(0,1)$. Then, the beliefs become $\mu_{B B}<\frac{1}{2}, \mu_{B A}<\frac{1}{2}$, and $\mu_{A B}>\frac{1}{2}$. The best responses of the receiver corresponding to these beliefs are $r_{B B}=1, r_{B A}=1$, and $r_{A B}=0$. The best responses of $S_{1}$ in turn become $p_{A}=0$ and $p_{B}=1$, which contradicts to our hypothesis.

Case 1.e: We next assume that $q_{A}(A)=q_{B}(A)=0$ and $q_{A}(B)=q_{B}(B)=1$. Then, the beliefs become $\mu_{B A}<\frac{1}{2}$ and $\mu_{A B}>\frac{1}{2}$. The best responses of the receiver corresponding to these beliefs are $r_{B A}=1$ and $r_{A B}=0$. The best responses of $S_{1}$ in turn become $p_{A}=0$ and $p_{B}=1$, which contradicts to our hypothesis.

Case 1.f: We next assume that $q_{A}(A)=q_{B}(A)=1$ and $q_{A}(B)=q_{B}(B) \in(0,1)$. Then, the beliefs become $\mu_{B B}<\frac{1}{2}, \mu_{B A}<\frac{1}{2}$, and $\mu_{A A}>\frac{1}{2}$. The best responses of the receiver corresponding to these beliefs are $r_{B B}=1, r_{B A}=1$, and $r_{A A}=0$. The best responses of $S_{1}$ in turn become $p_{A}=0$ and $p_{B}=1$, which provides a contradiction.

Case 1.g: We next assume that $q_{A}(A)=q_{B}(A)=1$ and $q_{A}(B)=q_{B}(B)=0$. Then, the beliefs become $\mu_{A A}>\frac{1}{2}$ and $\mu_{B B}<\frac{1}{2}$. The best responses of the receiver corresponding to these beliefs are $r_{A A}=0$ and $r_{B B}=1$. The best responses of $S_{1}$ again become $p_{A}=0$ and $p_{B}=1$, which is a contradiction.

The other symmetric cases give the similar contradictions, and thus, are omitted.

Case 2: We now consider that case where $S_{2}$ uses different strategies. Without loss of generality, we assume that $q_{A}(A)>q_{B}(A) \geq 0$ and $q_{A}(B)>q_{B}(B) \geq 0$. Again, suppose for a contradiction that $p_{A}>p_{B} \geq 0$. Then, the beliefs are calculated as $\mu_{A A}>\frac{1}{2}$ and $\mu_{B B}<\frac{1}{2}$. The corresponding best responses of the receiver at these information sets become $r_{A A}=0$ and $r_{B B}=1$. Note that for $q_{A}(A)>q_{B}(A)$ and $q_{A}(B)>q_{B}(B)$ to be part of an equilibrium, the receiver's strategies should satisfy $r_{A A} \geq r_{A B}$ and $r_{B A} \geq r_{B B}$. As $r_{A A}=0$ and $r_{B B}=1$, we get $r_{A B}=0$ and $r_{B B}=1$. Then, the best response of $S_{1}$ against the receiver's strategies become $p_{A}=0$ and $p_{B}=1$, which is the desired contradiction.

In the next step, we show that $S_{2}$ must use the same strategy in equilibrium, i.e., $q_{A}(A)=q_{B}(A)=$ $q_{1}$ and $q_{A}(B)=q_{B}(B)=q_{2}$. 
Case 1: We first assume that $S_{1}$ uses the same strategy.

Case 1.a: Suppose that $p_{A}=p_{B} \in(0,1)$. Assume for a contradiction that $q_{A}(A)>q_{B}(A)$. This implies $\mu_{A A}>\frac{1}{2}$ and $\mu_{A B}<\frac{1}{2}$. The best responses of the receiver in turn becomes $r_{A A}=0$ and $r_{A B}=1$. The best responses of $S_{2}$ against the receiver's strategy is $q_{A}(A)=0$ and $q_{B}(A)=1$, which contradicts to our hypothesis.

Case 1.b: Suppose that $p_{A}=p_{B}=0$. Assume for a contradiction, $q_{A}(B)>q_{B}(B)$. This implies $\mu_{B A}>\frac{1}{2}$ and $\mu_{B B}<\frac{1}{2}$. The best responses of the receiver in turn becomes $r_{B B}=1$ and $r_{B A}=0$. The best responses of $S_{2}$ against the receiver's strategy is $q_{A}(B)=0$ and $q_{B}(B)=1$, which contradicts to our hypothesis.

Case 1.c: Suppose that $p_{A}=p_{B}=1$. Assume for a contradiction, $q_{A}(A)>q_{B}(A)$. This implies $\mu_{A A}>\frac{1}{2}$ and $\mu_{A B}<\frac{1}{2}$. The best responses of the receiver in turn becomes $r_{A A}=0$ and $r_{A B}=1$. The best responses of $S_{2}$ against the receiver's strategy is $q_{B}(A)=1$ and $q_{A}(A)=0$, which contradicts to our hypothesis.

All other symmetric cases give the desired results.

Case 2: We now assume that $S_{1}$ uses different strategies; and without loss of generality assume $p_{A}>p_{B} \geq 0$. To arrive at a contradiction, without loss of generality, we assume that $q_{A}(A)>q_{B}(A)$ and $q_{A}(B)>q_{B}(B)$. The beliefs can be calculated as $\mu_{A A}>\frac{1}{2}$ and $\mu_{B B}<\frac{1}{2}$. The best responses of the receiver in turn becomes $r_{A A}=0$ and $r_{B B}=1$. For $q_{A}(A)>q_{B}(A)$ and $q_{A}(B)>q_{B}(B)$ to be a part of equilibrium, the receiver's equilibrium strategies should satisfy $r_{A A} \geq r_{A B}$ and $r_{B A} \geq r_{B B}$. As $r_{A A}=0$ and $r_{B B}=1$, we get $r_{A B}=0$ and $r_{B A}=1$. Against these strategies of the receiver, the best responses of $S_{1}$ satisfy $p_{A}=0$ and $p_{B}=1$, which contradicts to the hypothesis.

\section{References}

1. Crawford, V.; Sobel, J. Strategic information transmission. Econometrica 1982, 50, 1431-1451. [CrossRef]

2. Dickhaut, J.; McCabe, K.; Mukherji, A. An experimental study on strategic information transmission. Econ. Theory 1995, 6, 389-403. [CrossRef]

3. Cai, H.; Wang, J. Overcommunication in strategic information transmission games. Games Econ. Behav. 2006, 56, 7-36. [CrossRef]

4. Sánchez-Pagés, S.; Vorsatz, M. An experimental study of truth-telling in a sender receiver game. Games Econ. Behav. 2007, 61, 86-112. [CrossRef]

5. Sánchez-Pagés, S.; Vorsatz, M. Enjoy the silence: An experiment on truth-telling. Exp. Econ. 2009, 12, $220-241$. [CrossRef]

6. Sutter, M. Deception through telling the truth?! Experimental evidence from individuals and teams. Econ. J. 2009, 12, 220-241. [CrossRef]

7. Peeters, R.; Vorsatz, M.; Walz, M. Truth, trust, and sanctions: On institutional selection in sender-receiver games. Scand. J. Econ. 2013, 115, 508-548. [CrossRef]

8. Gurdal, M.Y.; Ozdogan, A.; Saglam, I. Truth-telling and trust in sender-receiver games with intervention: An experimental study. Rev. Econ. Des. 2014, 18, 83-103. [CrossRef]

9. Gilligan, T.; Krehbiel, K. Asymmetric information and legislative rules with a heterogeneous committee. Am. J. Political Sci. 1989, 33, 459-490. [CrossRef]

10. Krishna, V.; Morgan, J. A model of expertise. Q. J. Econ. 2001, 116, 747-775. [CrossRef]

11. Gick, W. Cheap Talk Equilibria-A Note on Two Senders; Mimeo, Harvard University: Cambridge, MA, USA, 2008.

12. Li, M. Two (talking) heads are not better than one. Econ. Bull. 2008, 3, 1-8.

13. Li, M. Advice from multiple experts: A comparison of simultaneous, sequential, and hierarchical communication. B.E. J. Theor. Econ. 2010, 10, 18. [CrossRef]

14. Austen-Smith, D. Information transmission in debate. Am. J. Political Sci. 1990, 34, 124-152. [CrossRef]

15. Austen-Smith, D. Credible debate equilibria. Soc. Choice Welf. 1990, 7, 75-93. [CrossRef]

16. Austen-Smith, D. Interested experts and policy advice: Multiple referrals under open rule. Games Econ. Behav. 1993, 5, 3-43. [CrossRef] 
17. Milgrom, P.; Roberts, J. Relying on information of interested parties. Rand J. Econ. 1986, 17, 18-32. [CrossRef]

18. Austen-Smith, D. Information acquisition and orthogonal argument, in Political Economy: Institutions Competition and Representation. In Proceedings of the Seventh International Symposium in Economic Theory and Econometrics; Barnet, W.A., Melvin, H.J., Schofield, N., Eds.; Cambridge University Press: Cambridge, UK; New York, NY, USA; Melbourne, Australia, 1993; pp. 407-436.

19. Battaglini, M. Multiple referrals and multidimensional cheap talk. Econometrica 2002, 70, 1379-1401. [CrossRef]

20. Battaglini, M. Policy advice with imperfectly informed experts. Adv. Theor. Econ. 2004, 4, 1. [CrossRef]

21. Ambrus, A.; Takahashi, S. Multi-sender cheap talk with restricted state spaces. Theor. Econ. 2008, 3, 1-27.

22. Lai, E.K.; Lim, W.; Wang, J.T.Y. Experimental Implementations and Robustness of Fully Revealing Equilibria in Multidimensional Cheap Talk; Mimeoy: Cambridge, MA, USA, 2011.

23. Minozzi, W.; Woon, J. Competition and Exaggeration in Experimental Cheap Talk Games. 2018 unpublished manuscript.

24. Vespa, E.; Wilson, A.J. Communication with multiple senders: An experiment. Quant. Econ. 2016, 7, 1-36. [CrossRef]

25. Peeters, R.; Vorsatz, M.; Walz, M. Rewards in an experimental sender-receiver game. Econ. Lett. 2008, 101, 148-150. [CrossRef]

26. Fischbacher, U. Z-Tree - Zurich toolbox for readymade economic experiments. Exp. Econ. 2007, 10, 171-178. [CrossRef]

(C) 2020 by the authors. Licensee MDPI, Basel, Switzerland. This article is an open access article distributed under the terms and conditions of the Creative Commons Attribution (CC BY) license (http:/ / creativecommons.org/licenses/by/4.0/). 